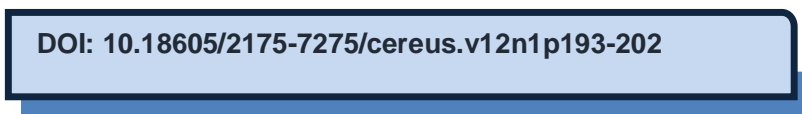

$<<$ Recebido em: 27/02/2019 Aceito em: 08/03/2020>>

\title{
EXPERIÊNCIA PATERNA COM O RECÉM-NASCIDO A PARTIR DAS ORIENTAÇÕES
}

DE ENFERMAGEM

\section{PATERNAL EXPERIENCE WITH THE NEWBORN FRON NURSING ORIENTATIONS}

Ricardo Cordeiro de Lima ${ }^{1}$, Ricardo Saraiva Aguiar²

\section{RESUMO}

Objetivo: descrever a experiência paterna com o recém-nascido a partir das orientações de enfermagem. Métodos: trata-se de uma pesquisa de campo de natureza exploratória com abordagem qualitativa realizada por meio de questionário semiestruturado. O projeto de pesquisa foi submetido ao Comitê de Ética em Pesquisa da Universidade Paulista - UNIP, sendo aprovado por meio do parecer $n^{\circ} \mathbf{2} .547 .105$ (CAAE 83687518.1.0000.5512). As respostas foram gravadas e transcritas na íntegra, sendo analisadas por meio da proposta de análise de conteúdo. Resultados: foram entrevistados 7 homens cujos filhos possuíam idade entre 1 a 4 meses de idade. Conclusão: os pais estiveram envolvidos de diversas maneiras durante a gestação de suas companheiras mostrando-se participativos. No entanto, alguns pais encontraram dificuldades para poder ofertar o cuidado continuado no lar à criança por falta de orientações dos profissionais de saúde. Diante deste fato, cabe a participação mais ativa do enfermeiro durante o pré-natal de modo a favorecer a inclusão dos pais nas atividades.

Palavras-chave: Paternidade. Saúde da Criança. Estratégia Saúde da Família. Enfermagem.

\section{ABSTRACT}

Objective: to describe the father's experience with the newborn from the nursing guidelines. Methods: this is an exploratory field research with a qualitative approach carried out through a semi-structured questionnaire. The research project was submitted to the Research Ethics Committee of Universidade Paulista - UNIP, and was approved by means of opinion No. 2.547.105 (CAAE 83687518.1.0000.5512). The responses were recorded and transcribed in full, being analyzed through the proposed content analysis. Results: 7 men were interviewed whose children were between 1 and 4 months old. Conclusion: the parents were involved in several ways during the gestation of their partners, showing themselves to be participative. However, some parents found it difficult to offer continued care at home to the child due to the lack of guidance from health professionals. Given this fact, it is up to the nurse's more active participation during prenatal care in order to favor the inclusion of parents in activities.

Keywords: Paternity. Child health. Family health strategy. Nursing.

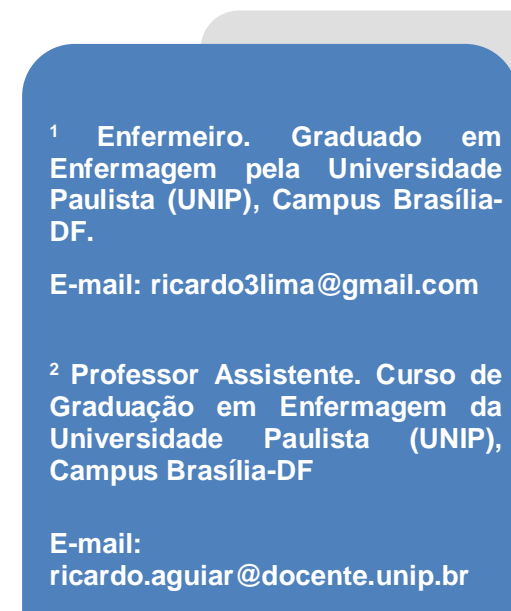

Enfermeiro. Graduado em DF.

E-mail: ricardo3lima@gmail.com

2 Professor Assistente. Curso de Graduação em Enfermagem da Universidade Paulista (UNIP), Campus Brasilia-DF

E-mail:

ricardo.aguiar@docente.unip.br

\section{INTRODUÇAO}

A alta hospitalar do recém-nascido $(\mathrm{RN})$ pressupõe o começo de uma nova etapa. $\mathrm{O}$ RN recebe alta quando seu estado de saúde permitir ou quando está em condições de ter um tratamento continuado em casa, sendo um momento esperado por toda a família, 
gerando um sentimento de conforto e alívio aos pais, porém é tempo de adaptações e cuidados (MARSKI et al., 2016; POMPEO et al., 2007; JAGER; BOTTOLI, 2011).

O RN na fase pós-natal, é visto como um ser ativo e essencialmente social. Assim, deve-se assegurar aos pais um papel importante na estruturação do desenvolvimento da criança, quando propício à construção de dependência e identificação para com o seu filho (POMPEO et al., 2007; JAGER; BOTTOLI, 2011).

Nessa perspectiva, a interação do homem nas etapas da gravidez vem aumentando em função da corresponsabilidade em promover o efetivo comprometimento com a paternidade consciente e mudanças sexuais impostas pelas conversões nos papeis dos genitores (FABBRO; LIMA, 2017; FREITAS; COELHO; SILVA, 2007; BALICA; AGUIAR, 2019). Diante disso, torna-se fundamental a participação do enfermeiro e dos demais profissionais de saúde na promoção dos sentimentos de competência e de confiança ao genitor, reforçando sua valiosa contribuição para a saúde de seu filho (NOGUEIRA; FERREIRA, 2012; AGUIAR; SANTANA; SANTANA, 2015).

Para tanto, os profissionais de saúde, prioritariamente os enfermeiros, devem conhecer os protocolos de cuidados dos nascidos a termo, manejo e as intervenções de enfermagem na orientação do pai com informações detalhadas e específicas ainda durante a assistência pré-natal (JAGER; BOTTOLI, 2011; SANTOS; KREITZ, 2014; NOGUEIRA; FERREIRA, 2014).

Nesse contexto, percebe-se a importância dos profissionais de saúde que atuam na atenção ao pré-natal, proporcionando aos pais orientações básicas e específicas a respeito do $\mathrm{RN}$ e lactente neste período. Trata-se de uma abordagem educativa e preventiva com finalidade de acompanhar o genitor oferecendo apoio emocional, informações para o plano de alta e questões complexas que podem surgir no período do retorno do RN ao lar (SANTOS; KREITZ, 2014; NOGUEIRA; FERREIRA, 2014; AGUIAR et al., 2013).

Assim, este trabalho poderá contribuir para a razão da necessidade de investigação científica quanto ao acolhimento paterno, pois o sucesso no atendimento está relacionado com a avaliação das mudanças que se operam nas suas condições culturais, sociais e com o envolvimento da equipe com as práticas auxiliares, tornando mais brandos possíveis obstáculos a serem superados nesta fase da vida, que requer ações e orientações por parte da equipe para posterior envolvimento dos pais.

Portanto, este trabalho tem o objetivo de descrever a experiência paterna com o recém-nascido a partir das orientações de enfermagem. 


\section{MATERIAIS E METODOS}

Trata-se de uma pesquisa de campo de natureza exploratória com abordagem qualitativa.

A pesquisa foi realizada em uma Unidade Básica de Saúde (UBS) da região administrativa de Samambaia, Distrito Federal (DF).

Os sujeitos da pesquisa foram homens com filhos nascidos vivos e que estavam na UBS acompanhando o atendimento de saúde de seus filhos no momento da coleta de dados. A determinação do número de participantes obedeceu ao critério de saturação das informações. Ao final da coleta de dados, contou-se com a participação de sete pais que responderam sobre a experiência paterna com o lactente por meio de uma entrevista semiestruturada.

Todos os entrevistados assinaram o Termo de Consentimento Livre e Esclarecido (TCLE). O projeto de pesquisa foi submetido ao Comitê de Ética em Pesquisa da Universidade Paulista (UNIP), sendo aprovado por meio do parecer nº 2.547.105 (CAAE 83687518.1.0000.5512), no intuito do cumprimento das diretrizes da Resolução no 466/2012 do Conselho Nacional de Saúde (CNS) no que se refere à pesquisa com seres humanos.

Os sujeitos do estudo respeitaram os seguintes critérios de inclusão: homens com idade maior ou igual a 18 anos, de qualquer etnia, escolaridade, desde que tenham filhos lactentes vivos.

As entrevistas foram aplicadas de forma individual e presencial. Foram solicitadas algumas respostas às perguntas referentes ao tema de pesquisa, deixando os pesquisados livres para falar sobre o assunto.

Para a análise do conteúdo temático das informações obtidas com os sujeitos de pesquisa, foi seguida a ordem das respostas dos pais que foram classificados como P1, P2, P3, P4, P5, P6 e P7, podendo se adequar posteriormente.

As respostas foram analisadas por meio da proposta de Análise de Conteúdo, a saber: categorização, inferência, descrição e interpretação.

\section{RESULTADOS E DISCUSSAO}

Os dados sócio-demográficos obtidos por meio do questionário semiestruturado aplicados aos pais dos lactentes demonstrou que as crianças tinham idade entre 1 a 4 
meses de vida; 2 homens consideram sua cor como branca, 1 negro e 04 pardos; a idade dos entrevistados variou de 18 a 35 anos, onde 2 homens não quiseram informar a idade.

Dos 7 entrevistados, 6 estavam casados ou vivendo com companheira e 1 entrevistado era solteiro; os 7 estavam empregados, 3 com renda familiar entre $R \$ 800,00$ a 2.500,00, 2 mencionaram que a sua renda era de $R \$ 4.000,00,1$ era $R \$ 10.000,00$ e 1 não quis informar o valor da sua renda; a respeito do nível de escolaridade foi identificado que 1 tinha o ensino fundamental, 2 o ensino médio completo, 10 ensino médio incompleto, 2 o ensino superior completo e 1 tinha ensino superior incompleto.

Quanto a quantidade de filhos, 3 pais responderam que tinham apenas 1 filho, 1 pai respondeu que tinha 2 filhos, 1 pai tinha 3 filhos e 2 pais tinham 04 filhos.

\section{Categoria1: Apoio paterno durante o período pós-parto e vínculos paternos}

A importância do acompanhamento paterno durante a gestação é vista como uma prática que proporciona uma gravidez tranquila gerando assim a criação de vínculos entre o pai e o bebê (TEIXEIRA et al., 2014; PICCININI et al., 2004). Para o homem a importância do acompanhamento paterno durante a gestação envolve sentimentos e emoções positivas o que pode ser identificado nas falas a seguir:

Muito importante. Uma gravidez tranquila com apoio do pai faz com que a mãe e o bebê depois de nascido tenham uma relação mais confortável e calma. (P6)

Para conscientização e criação de vínculos paternos. (P7)

Ela em momento algum pode ser descartada, pois a paternidade tem muita influência no período de resguardo. O pai deve estar presente. (P5)

A importância da obtenção do afeto paterno durante o período pós-parto possibilita o bem-estar na companheira, trazendo consigo o conforto e o desenvolvimento da saúde do filho durante a gestação. É de grande importância ter o reconhecimento da igualdade nas relações de gênero colocando em prática os direitos e deveres da vivência familiar, isso tudo para garantir que as experiências afetivas da paternidade possam ser vividas de forma saudável (TEIXEIRA et al., 2014).

A presença cuidadosa do homem na gravidez, no nascimento e no período pósnascimento possibilita a geração e a construção de vínculos paternos afetuosos e que podem proporcionar o bem-estar com a criança e a todos os envolvidos, além de 
estabelecer um relacionamento saudável e a garantia de uma melhor qualidade de vida para a família e seus membros. A experiência adquirida na paternidade é marcada como satisfatória pelo conhecimento adquirido durante a gestação, proporcionando ao pai crescimento pessoal e sentimento de autorrealização e, principalmente do privilégio de ser pai (TEIXEIRA et al., 2014).

A necessidade da influência da paternidade no período do pós-parto é de grande importância na interação entre pai-filho, sendo um dos fatores decisivos para 0 desenvolvimento de vínculos paternos, surgindo possibilidades de construção psicoafetiva - fundamental no processo de desenvolvimento da criança -, e auxiliando nas dificuldades próprias a este período (PICCININI et al., 2004).

Baseado nisso, a gestação pode ser compreendida como um momento de preparação psicológica para a paternidade, originando uma fase de importantes reestruturações na vida da mulher e do homem e nos papéis que estes exercem. A partir do momento em que se inicia uma gravidez, algumas atitudes devem ser tomadas, como por exemplo, a inserção do pai nas consultas de pré-natal.

\section{Categoria 2: Apoio paterno}

É possível verificar que os pais possuem conhecimentos acerca do papel deles no apoio psicológico e no incentivo da amamentação:

Fundamental e ajuda a mãe. (P1)

O pai deve ser prestativo, ajudar a mulher com as coisas de casa e também ajudar com o recém-nascido, pois não é apenas papel da mãe. (P5)

Cuidar da criança, bem como da mãe. (P2)

Ajudar nas tarefas domésticas, apoio psicológico e incentivar amamentação. (P6)

A falta de apoio oferecido pelo parceiro no período pós-parto pode resultar em um transtorno depressivo, provido pela a falta de atenção do pai e apoio nos cuidados com o recém-nascido. Vale ressaltar que, muitas vezes, é difícil haver o reconhecimento do pai quanto à fragilidade da esposa, o cansaço acentuado devido às noites mal dormidas, diminuição da autoestima e a falta do desejo sexual. No entanto, acredita-se que o apoio do parceiro, de familiares e amigos possa ser um fator protetor para o aparecimento de tais consequências negativas da depressão materna (OLIVEIRA; BRITO, 2009). 
Nessa perspectiva, o período de alta hospitalar do RN é uma fase no qual deve haver cuidado com a mãe e com a criança, pois o cuidar é uma ação de envolvimento afetivo um com o outro e a figura paterna traz o reconhecimento em que a alta hospitalar é uma fase que requer cuidados, prezando pelo bem-estar da companheira e de seu filho (OLIVEIRA; BRAGA, 2016; RIBEIRO et al., 2015).

Assim, a prática do cuidado requer conhecimento do ser cuidado. O cuidador deve ser capaz de entender as necessidades do outro e responder a elas de forma adequada e em uma relação que fortaleça a do outro. Diante disso, pode-se dizer que a ação do pai é determinada pelo resultado da interação dele consigo mesmo, com sua companheira, filho e ambiente no qual está inserido. Sua atitude de cuidar é construída em resposta ao significado que a esposa e o filho têm para ele como partes do seu núcleo familiar (RIBEIRO et al., 2015).

\section{Categoria 3: Informações aos pais sobre os recém-nascidos}

A pesquisa identificou que ainda há falta de orientação aos pais pelos profissionais de saúde sobre os cuidados com o filho após a alta hospitalar:

Muito pouco. Acho que deveria ser mais bem informado o pai de primeira viagem. $(P 1)$

Não. Nenhum profissional [...] me passou informação. (P3)

Não. (P4)

Sim. Fizemos alguns cursos, consultorias, livros, vídeos e até orientação no posto de saúde. (P6)

A realidade sobre o cuidado da criança após a alta hospitalar é exposta pela compreensão paterna após a chegada do $\mathrm{RN}$ ao domicilio se tornando um período de adaptações. É o momento em que o conhecimento adquirido sobre os cuidados com o RN se transforma de fato nas intervenções que deverão ser tomadas no domicilio (RIBEIRO et al., 2015; FREITAS; COELHO; SILVA, 2007; FABBRO; LIMA, 2017).

Assim, os pais devem ser esclarecidos quanto ao processo de adaptação e chegada da criança. A aprendizagem prévia dos pais os prepara para a chegada do filho a partir de informações sobre as atitudes e cuidados que possam ajudar sua companheira e seu filho. Muitas vezes, fica notório a angústia emocional e o desespero do pai em relação aos 
conhecimentos específicos que geram em se ter uma criança necessitando de cuidado e atenção (BRAMBILA et al., 2015).

Baseado nisso, é fundamental a capacitação do pai para a prestação de cuidados. Para se obter estratégias que visem alcançar resultados desejáveis para a promoção de saúde ao recém-nascido, o enfermeiro deve envolver a execução de um adequado plano de educação para a saúde no qual deverá ofertar orientações que irão prover ao pai conforto e segurança perante ao recém-nascido (ROQUE; COSTA, 2014).

O acontecimento da alta hospitalar representa um período em que se traz insegurança e dúvidas. Mesmo que os pais estejam capacitados a partir desse momento, eles se encontram sozinhos na responsabilidade de ofertar com segurança e conforto o cuidado com o filho desempenhando o seu papel de pai e de mãe. Assim, se faz de grande importância certificar a competência dos pais nos cuidados ao RN durante a internação, compreendendo o propósito da redução da ansiedade e a melhoria da autoconfiança, preparando-os para a alta através dos ensinos em um processo constante de educação para a saúde (CASTOLDI; RIBEIRO; SOBREIRA, 2014).

Assim, a necessidade da construção de um plano de alta se faz importante ao longo do tempo de internação, onde se deve implicar tanto os profissionais de saúde e os familiares responsáveis pelo RN. Além disso, deve-se garantir o retorno dessa família para o acompanhamento na Estratégia de Saúde da Família (ESF) (LOPES; AGUIAR, 2020).

\section{Categoria 4: Amor paterno no período gestacional e participação ativa dos pais nas consultas de pré-natal}

A experiência paterna adquirida durante o período gestacional pode ajudar com os cuidados com o RN, garantindo o crescimento e desenvolvimento saudável da criança e provendo o vínculo afetivo entre o pai e a criança (FREITAS; COELHO; SILVA, 2007), como percebe-se nos depoimentos a seguir:

Pode sim. O amor que cria no período gestacional é passado para a criança: a atenção, o cuidado. (P5)

Acho que sim! Pela participação ativa nas consultas, acabei recebendo muitas informações uteis. (P7) 
Pôde-se verificar que os pais participam de inúmeras formas na gravidez de suas companheiras, tanto de modo direto, através de comportamentos como acompanhar as ecografias e consultas, como de modo indireto, oferecendo-se como uma figura de apoio para a gestante e expressando o seu envolvimento emocional. O contato visual que se dá com o feto através da ecografia desencadeia nos pais reações positivas que proporcionam uma sensação de presença concreta do bebê e isso pode encorajar um maior interesse e envolvimento dos pais com o filho, constituindo-se um indicativo do envolvimento emocional paterno durante a gestação (ROQUE; COSTA, 2014; CASTOLDI; RIBEIRO; SOBREIRA, 2014).

O período gestacional age como forma de preparo para novos papeis que surgirão, fazendo de forma particular que podem ser compreendido através do vínculo entre pai e filho. A responsabilidade paterna durante a gestação não se refere apenas em ações como o acompanhamento nas consultas, mas sim na compreensão e participação de atividades relacionadas às gestantes como a preparação para a chegada do bebê e o suporte emocional (FABBRO; LIMA, 2017).

Durante este seguimento o homem conquista aos poucos novas capacidades que representam o desenvolvimento saudável do filho, ou seja, na proporção que o filho cresce o homem se constrói como pai. Portanto, no período da gestação como do nascimento, a construção da paternidade se torna sensível pelas mudanças que ocorrem (RIBEIRO et al., 2015).

\section{CONSIDERAÇOES FINAIS}

Este estudo permitiu descrever a experiência paterna com o $\mathrm{RN}$, destacando-se a importância que a vida tem para todos os profissionais de saúde, em particular o enfermeiro proporcionando atendimento digno aos genitores. Esclarecer possíveis dúvidas, através de uma boa e adequada assistência de enfermagem deve fazer parte do atendimento humanizado que deve ser prestado a todos os pacientes.

O objetivo deste estudo foi descrever a experiência paterna com o recém-nascido no que se refere ao cuidado, onde os pais expressaram opiniões positivas em relação à experiência paterna, podendo assim estar preparados para adaptações que possam proporcionar condições de conforto e alívio à criança.

Os pais estiveram envolvidos de diversas maneiras durante a gestação de suas companheiras, mostrando-se participativos. No entanto, alguns pais encontraram 
dificuldades para poder ofertar o cuidado continuado no lar à criança por falta de orientações dos profissionais de saúde. Diante deste fato, cabe a participação mais ativa do enfermeiro durante o pré-natal de modo a favorecer a inclusão dos pais nas atividades.

Portanto, esta pesquisa poderá contribuir para a razão da necessidade de investigação cientifica quanto ao acolhimento paterno.

\section{REFERENCIAS}

AGUIAR, R. S. et al. Percepção de mulheres sobre o acolhimento oferecido pelo enfermeiro no pré-natal. Cogitare enferm., v. 18, n. 4, p. 756-60, 2013.

AGUIAR, R. S.; SANTANA, D. C.; SANTANA, P. C. A percepção do enfermeiro da estratégia saúde da família sobre a saúde do homem. Rev. enferm. Cent.-Oeste. Min., v. 5, n. 3, p. 1844-54, 2015.

BALICA, L. O.; AGUIAR, R. S. Percepções paternas no acompanhamento pré-natal. Rev. aten. saúde, v. 17, n. 61, p. 114-26, 2019.

BRAMBILA, I. L. M. et al. O cuidado domiciliar ao recém-nascido de risco no primeiro ano de vida: experiência dos pais. Diálogos \& Saberes, v. 11, n. 1, p. 73-92, 2015.

CASTOLDI, L.; RIBEIRO, T. G.; SOBREIRA, R. D. C. L. Envolvimento paterno da gestação ao primeiro ano de vida do bebê. Psicol. Estud., v. 19, n. 2, p. 247-59, 2014.

FABBRO, M. R. C.; LIMA, M. P. A experiência paterna de pais de "primeira viagem" no cuidado do bebê nos primeiros três meses de vida. Atas CIAIQ2017, v. 2, n. 1, p. 166-75, 2017.

FREITAS, W. M. F.; COELHO, E. A. C.; SILVA, A. T. M. C. Sentir-se pai: a vivência masculina sob o olhar de gênero. Cad. Saúde Pública, v. 23, n. 1, p. 137-145, 2007.

JAGER, M. E.; BOTTOLI, C. Paternidade: vivência do primeiro filho e mudanças familiares. Psicol. teor. prat., v. 33, n. 1, p. 141-53, 2011.

LOPES, L. C. S.; AGUIAR, R. S. Aplicabilidade das boas práticas de atenção ao parti: revisão integrativa de literatura. REVISA, v. 9, n. 1, p. 133-43, 2020.

MARSKI, B. S. L. et al. Alta hospitalar do recém-nascido prematuro: experiência do pai. Rev. bras. enferm., v. 69, n. 2, p. 202-9, 2016.

NOGUEIRA, J. R. D. F.; FERREIRA, M. O envolvimento do pai na gravidez/parto e a ligação emocional com o bebê. Referência, v. 3, n. 8, p. 57-66, 2012.

OLIVEIRA, E. M. F.; BRITO, R. S. Ações de cuidado desempenhadas pelo pai no puerpério. Esc. Anna Nery Rev. enferm., v. 13, n. 3, p. 595-601, 2009. 
OLIVEIRA, A. P.; BRAGA, T. L. Depressão pós-parto: consequências para mãe e o recém-nascido: uma revisão sistemática. Revista Eletrônica Estácio Saúde, v. 5, n. 1, p. 133-44, 2016.

PICCININI, C. A. et al. O envolvimento paterno durante a Gestação. Psicol. reflex. crit., v. 17, n. 3, p. 303-14, 2004.

POMPEO, A. D. et al. Atuação do enfermeiro na alta hospitalar: reflexões a partir dos relatos de pacientes. Acta Paul. Enferm., v. 20, n. 3, p. 345-50, 2007.

RIBEIRO, J. P. et al. Participação do pai na gestação, parto e puerpério: refletindo as interfaces da assistência de enfermagem. Espaço para a saúde, v. 16, n. 3, p. 73-82, 2015.

ROQUE, S. S. G.; COSTA, M. G. F. A. Preparação dos pais para o cuidar do recémnascido após a alta: avaliação dos registros de enfermagem. Millenium, v. 47, n. 1, p. 4760, 2014.

SANTOS, S. C.; KREUTZ, C. M. O envolvimento do pai na gestação do primeiro filho. Pensando fam., v. 18, n. 2, p. 62-76, 2014.

TEIXEIRA, R. C. et al. Vivências e necessidades de saúde de homens no período pósnascimento de um filho. Rev. bras. enferm., v. 67, n. 5, p. 780-7, 2014. 